\title{
Research on manufacturability evaluation system based on features for automobile gears
}

\author{
Wang Yao ${ }^{1, a}$, Hu Yanjuan ${ }^{2, b^{*}}$, Shi Chao ${ }^{2, c}$ \\ ${ }^{1}$ College of Mechanical Engineering, Beihua University, Jilin 132021, China \\ ${ }^{2}$ School of Techtronic Engineering, Changchun University of Technology, Changchun 130012, \\ China \\ awywy7731@126.com, byanjuan_hu@126.com, ${ }^{\mathrm{a}}$ 645519398@qq.com \\ *Corresponding author: Hu Yanjuan(1980-), yanjuan_hu@126.com
}

\begin{abstract}
Keywords: manufacturability evaluation; feature; automobile gear; system.
Abstract. Aiming at automobile gears, the three-level manufacturability evaluation system was proposed. The system includes the structural processing evaluation, the machinability evaluation of features and comprehensive evaluation. This paper mainly introduces the first and second level manufacturability evaluation. And the example proves the effectiveness of the system.
\end{abstract}

\section{Introduction}

Since the1990s, the rapid development of information technology, to speed up the transfer of knowledge, to improve every aspect of the frequency of updates, manufacturing system is facing rapid changes in two aspects of external environment and internal environment. On the external environment, manufacturing system must adapt to the social and economic environment for the development of requirements, such as manufacturing activity globalization, green manufacturing, reduce consumption, energy and raw material products, etc. "The first technology and personalization," global knowledge economy marked the product competition has been from the original price competition into the competition of the technical content, and the technological content of products become the mainstream in the market and the key to the success of enterprise[1,2]. The customers on the performance of the product, specifications, and varieties put forward new requirements, plural, individuality, more variety, and small batch production become the dominant form; in the meantime, the rapidly changing and increasingly fierce market competition in the manufacturing industry has proposed a more demanding TQCS (T time-to-market, Q product quality, C low-cost, high-value-added, S service satisfaction) requirements, the sustainable development strategy also requires the negative impact manufacturing environment is minimized. The virtual manufacturing technology can effectively solve these problems, it is the premise of simulation technology, it can form a virtual environment, a virtual manufacturing process, a virtual products, a virtual businesses, thereby greatly shorten product development cycles, improve the success rate[3]. The manufacturability evaluation is important part of the virtual manufacturing technology, which can be pre-evaluate and predict product manufacturability[4].

\section{Research on characteristics of the manufacturability evaluation system}

Manufacturability of part, which includes two meanings: the first refers to the feasibility of the design process of part, and the second is from the point of view of manufacturing resources to measure the degree of difficulty in some parts manufacturing. Such as the part structure is whether reasonable, and the part characteristics are whether compatible, size marking is with no error, tolerance design is consistent, all those are as the first level meaning, and manufacturing issues in this level do not refer to specific manufacturing resources, that kind of problem cannot be processed in any manufacturing resources environment. As for manufacturing resources for capacity of the processing part, as well as the process for reasonable economic, which belong manufacturability evaluation of the second layer meaning. The level of manufacturability problem is related to the specific manufacturing resources. Manufacturability evaluation refers to the current manufacturing resources, the machining 
characteristics and the design of parts required to meet the level of manufacturing resource constraints analysis to identify part design and not conducive to the manufacture of parts to ensure quality factors, and feedback to the design staff in order to improve the design.

Manufacturability evaluation system in this paper is composed of the structural processing evaluation, machinability and integrated evaluation. It can drill in favor of early detection evaluate a design problem in reducing the time and complexity of manufacturability evaluation. The third-level manufacturability evaluation architecture feature-based is shown in Fig. 1.

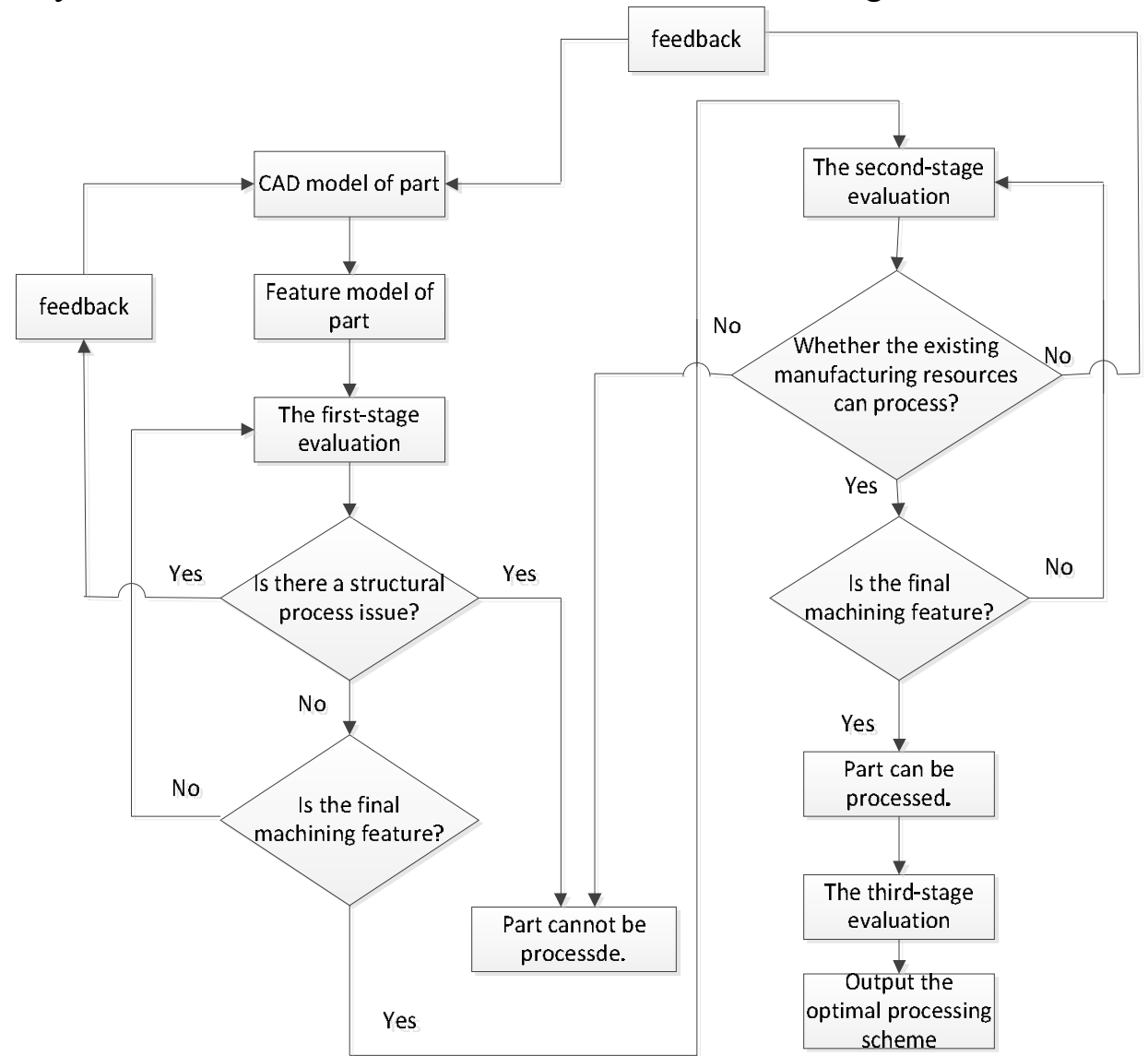

Fig.1 Manufacturability evaluation system

The first-level evaluation process is a structural processing evaluation, which is mainly structural review and evaluate whether there are structural problems for the machining process. If the structural issues exist, it cannot manufacture parts and ends manufacturability evaluation and reflects issue to the designer.

The second-level evaluation is a machinability evaluation, and it is primarily the evaluation of manufacturing resources and processing capabilities, which includes a feature processing capacity and quality of processing capacity in two ways. Parts of the second stage evaluation process to verify features in the existing manufacturing resources processed out whether the conditions, as well as parts of the design requirements can be guaranteed. If that cannot be authenticated, the parts in the conditions of existing manufacturing resources cannot be manufactured, will not be manufactured by machining features or design requirements feed back to the designer. If that is verified, it will output the evaluation results that parts can be manufactured, and the information technology equipment, machine tools and machining characteristics corresponding to each record.

The third-level evaluation is a comprehensive evaluation, as the manufacturing technology development, a part is often has multiple processing environments and processing schemes. Comprehensive Evaluation of the efficiency, quality, cost, and profits, perspective, and from more than one factory environment, select the most suitable for the processing environment, and from variety of processing program, the best of the processing program. 


\section{Example}

For the manufacture of the two stages of the main evaluation of the design of the parts in the existing manufacturing resources, and the environment, whether it can be processed. From a structural process rules, and manufacturing resources, including machine tools, tools, clamps, and measure tools and other constraints departure, for the manufacture evaluation. Taking the four-grade intermediate shaft gear for example as shown in Fig. 2, the evaluation can be carried out.

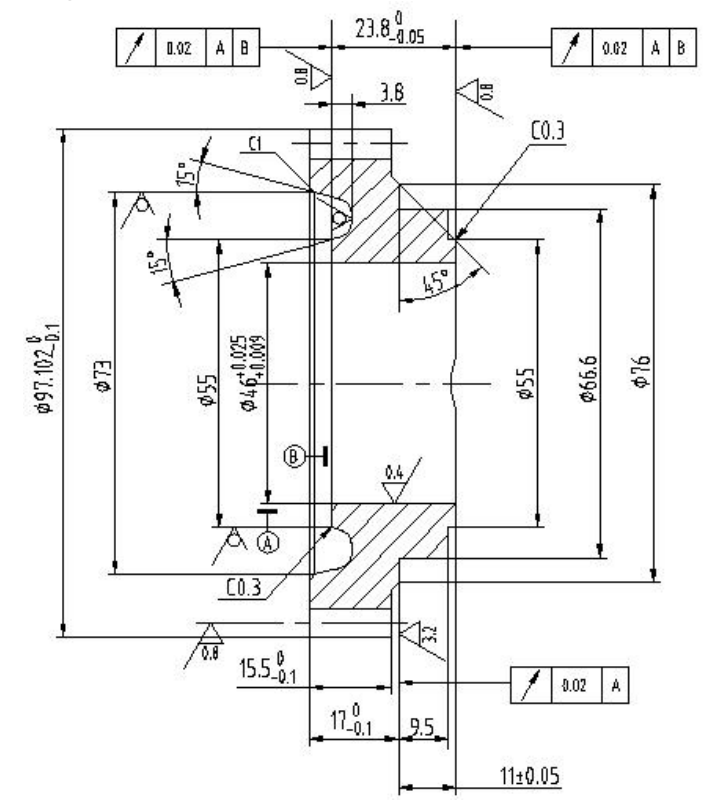

Fig. 2 The four-grade intermediate shaft gear

The first-stage evaluation should follow the structure rules, as well as this document combines automotive gear, it verifies that the gear does not have structure technology issues.

The second-level evaluation is a machinability evaluation, it can evaluate whether the existing manufacturing resources to meet the gear parts of the design and processing requirements. For example the constraints to the machine tool, all of the machine model information and to evaluate the existing processing equipment processing capacity. According to the apparent precision of the gear is required to achieve the seven finishing accuracy. The machinability evaluation test whether existing manufacturing resources can process the parts, as long as the existing processing equipment can meet the accuracy requirements of this part, the parts can be processed. According gear characteristics and processing capacity category search out relevant gear processing machine tools, such as CNC worm wheel gear grinding machine (processing range: the diameter 20 to $320 \mathrm{~mm}$, the modulus $1 \sim 6 \mathrm{MM}$, the tooth number $12 \sim 260$, the grinding accuracy $4 \sim 5$ level/GB 10,095 - 88) to meet the gear's accuracy requirements. Find the meet processing requirements of the machine, verify that you have to meet part processing requirements of the tool, clamp, and equipment to match the machine, if the output results of the assessment, that is the part in the existing manufacturing resources, it can be processed.

\section{Conclusions}

This paper established the three level manufacturability system based on feature. The first-level evaluation process is a structural processing evaluation, and it is mainly for process review according to the structure rules of the parts. The second-level evaluation is machinability evaluation, it checks the parts in the existing manufacturing resources environment whether can be processed, which is mainly from the machine tool constraint, the constraint tools, clamps constraint and other resource constraints set out to evaluate whether the processing capacity of these resources and meet parts of the design and processing requirements. The third-level evaluation is comprehensive evaluation, and from more than one factory environment, selecting the most suitable for the processing environment, and from variety of processing program, selecting the best of the processing program. The two level evaluations are mainly based on rules for qualitative assessment to determine whether or not the part can be processed, 
and to record all of the manufacturing resources which meet part processing requirements in order to carry out the next comprehensive evaluation.

\section{Acknowledgment}

This research work was supported by the Nature Science Foundation of China, and the project name is "Research on theory and method of manufacturability evaluation in cloud manufacturing environment", no. 51405030. The education department project of Jilin Province, no. [2014] 483.

\section{References}

[1] Ng A H C, Adolfsson J, Sundberg M, et al. Virtual manufacturing for press line monitoring and diagnostics. International Journal of Machine Tools \& Manufacture, 48(2008) 565-575.

[2]O. Kerbrat, P. Mognol, J.-Y. Hascoet. Manufacturing complexity evaluation at the design stage for both machining and layered manufacturing. CIRP Journal of Manufacturing Science and Technology, 2(2010) 208-215.

[3] B. Babic, N. Nesic, Z.Miljkovic. A review of automated feature recognition with rule-based pattern recognition. Computers in Industry, 59(2008)321-337.

[4] P. Vichare, A. Nassehi, S. Kumar, et al. A Unified Manufacturing Resource Model for representing CNC machining systems. Robotics and Computer-Integrated Manufacturing. 25(2009)999-1007. 